\title{
Qualidade e subjetividade na avaliação de programas e serviços em saúde
}

\author{
Quality and subjectivity in the evaluation \\ of health services and programs
}

Kátia Yumi Uchimura 1

Maria Lúcia Magalhães Bosi 2

\footnotetext{
1 Faculdade de Medicina, Universidade Federal do Ceará.

Rua Professor Costa Mendes 1608, Fortaleza, CE 60431-140, Brasil. katyumi@terra.com.br 2 Núcleo de Estudos em Saúde Coletiva, Universidade Federal do Rio de Janeiro. Av. Brigadeiro Trompowsky $s / n$, Bloco K, Rio de Janeiro, $R J$ 21944-970, Brasil. malubosi@nesc.ufrj.br
}

\begin{abstract}
This essay focuses on the evaluation of health programs and services, emphasizing the subjectivity emerging from the evaluation process. The text is a theoretical construction that targets the various meanings of evaluation, the influence of the positivist paradigm on the field of health program and service evaluation, the polysemous nature of the term "quality", and its close overlapping with subjectivity. In addition, the authors highlight the importance of studies that incorporate the perspectives of social actors into the evaluation processes, recommending qualitative social research methodology as a productive instrument.

Key words Evaluation; Health Services; Qualitative Analysis

Resumo Este ensaio versa sobre a avaliação de programas e serviços de saúde, dando ênfase à subjetividade imanente ao processo de avaliar. Trata-se de uma construção teórica que focaliza os diversos sentidos da avaliação, a influência do paradigma positivista no campo da avaliação de programas e serviços de saúde, o caráter polissêmico do termo qualidade e sua estreita imbricação com a subjetividade. Em adição, procuramos indicar a importância dos estudos que incorporam as perspectivas dos atores sociais nos processos de avaliação, apontando a metodologia qualitativa de pesquisa social como sendo um profícuo instrumento.

Palavras-chave Avaliação; Serviços de Saúde; Análise Qualitativa
\end{abstract}




\section{Introdução}

A avaliação representa uma atividade bastante antiga, presente desde os primórdios na história da humanidade (Contandriopoulos et al., 1997). Já o conceito de avaliação de programas públicos surge no cenário mundial logo após a Segunda Grande Guerra, em virtude da necessidade de melhoria da eficácia da aplicação dos recursos pelo Estado. Para essa finalidade, foram desenvolvidos inúmeros métodos a fim de possibilitar a análise das vantagens e dos custos de programas.

No Brasil, a pesquisa de avaliação de políticas começa a se desenvolver apenas a partir da década de 80 e apresenta-se, tanto do ponto de vista acadêmico (Rus-Perez, 1998), como da sua incorporação ao cotidiano da administração pública (Cotta, 1998), ainda bastante incipiente. Nesse âmbito, a avaliação tem sido conceituada sob diferentes perspectivas. Essas concepções guardam ora traços coincidentes ou comuns, ora posições discordantes.

Assim, na tipologia da avaliação, observa-se uma grande diversidade de abordagens incluindo modalidades classificatórias cuja relevância se manifesta em vista de seu interesse prático: de acordo com o momento da avaliação, a função da avaliação, a procedência dos avaliadores e, por fim, os aspectos do programa que são objeto da avaliação (Aguilar \& Ander-Egg, 1994).

Outras propostas, dentre elas a de Cohen \& Franco (1998), ratificam parcialmente tais critérios, em especial no que concerne ao momento e ao objetivo da avaliação e à procedência dos avaliadores, mas acrescentam critérios referentes à escala (magnitude) dos projetos e aos destinatários da avaliação. Revela-se, portanto, um vasto espectro de configurações e racionalidades.

Embora a avaliação possa assumir inúmeros matizes em consonância com diferentes perspectivas, nossa incursão pelo tema não tem o propósito de analisar toda a diversidade de abordagens referida na literatura. Nosso objetivo, ao percorrer o debate teórico sobre a temática, consiste em apontar os aspectos subjetivos inerentes à avaliação, especialmente quando a qualidade ocupa posição de destaque nesse processo.

\section{O campo da avaliação de serviços e programas}

A palavra avaliação, no sentido mais geral, consiste em atribuir valor a algo (Aguilar \& Ander-Egg, 1994). Nesta acepção, o termo avaliação refere-se ao ato ou efeito de avaliar (Ferrei- ra, 1986), ou seja, conferir valor, manifestar-se em relação a alguma coisa, sem compromisso, no entanto, com o fundamento desse juízo ou com um método específico.

Quando se trata, contudo, de avaliar serviços ou programas, o que corresponde a situar a avaliação no campo das modalidades de intervenção social e, conseqüentemente, das ciências sociais, recorre-se à utilização de "procedimentos que, apoiados no uso do método científico, servem para identificar, obter e proporcionar a informação pertinente e julgar o mérito e o valor de algo de maneira justificável" - a chamada avaliação em sentido estrito ou avaliação sistemática (Aguilar \& Ander-Egg, 1994:23).

No entendimento dos autores, não se evidencia uma diferença de objetivos ou propósitos, mas uma diferença de métodos entre as duas acepções. Nesse sentido, a avaliação sistemática pressupõe a utilização de um método, diferente da avaliação de fatos cotidianos, em que - baseados em determinados critérios de valor - são emitidos julgamentos de forma despreocupada. Poderíamos - grosso modo afirmar que significaria revestir com cientificidade a avaliação concebida no sentido lato para dar origem à pesquisa avaliativa, que se utiliza de métodos e técnicas da pesquisa social com a finalidade de possibilitar a emissão de um juízo com diligência, ou ainda, a realização de uma avaliação conseqüente.

Contandriopoulos et al. (1997:31) desenvolvem uma elaboração sobre o tema que reforça em parte essa idéia, mas consideram ser apenas parcial a correspondência entre a área da avaliação e a área da pesquisa. Para esses autores: "avaliar consiste fundamentalmente em fazer um julgamento de valor a respeito de uma intervenção ou sobre qualquer um de seus componentes, com o objetivo de ajudar na tomada de decisões. Este julgamento pode ser resultado da aplicação de critérios e de normas (avaliação normativa) ou se elaborar a partir de um procedimento científico (pesquisa avaliativa)".

Nessa perspectiva, a avaliação normativa é definida como “...a atividade que consiste em fazer um julgamento sobre uma intervenção, comparando os recursos empregados e sua organização (estrutura), os serviços ou os bens produzidos (processo), e os resultados obtidos, com critérios e normas" (Contandriopoulos et al., 1997:34); e a pesquisa avaliativa “...como o procedimento que consiste em fazer um julgamento ex-post de uma intervenção usando métodos científicos. Mais precisamente, trata-se de analisar a pertinência, os fundamentos teóricos, a produtividade, os efeitos e o rendimento de uma intervenção, assim como as relações exis- 
tentes entre a intervenção e contexto no qual ela se situa..." (Contandriopoulos et al., 1997:37).

A pesquisa avaliativa, de acordo com Silva \& Formigli (1994), é desenvolvida com base em desenhos que se "superpõem parcialmente" àqueles desenvolvidos pela epidemiologia, e, tal como se apresenta na proposta de Contandriopoulos, poderia ainda se desdobrar em seis tipos de análise, as quais devem ser contempladas no todo ou em parte, em se tratando de realizar uma pesquisa dessa natureza. Já à avaliação normativa, restaria a apreciação da estrutura, do processo (nas dimensões técnica, organizacional e das relações interpessoais) e dos resultados (Contandriopoulos et al., 1997).

Considerando que as análises que abordam a estrutura, o processo e/ou o resultado - propostas inicialmente por Donabedian em 1966 (apud Aguilar \& Ander-Egg, 1994) para a avaliação da qualidade de serviços de saúde e ainda hoje bastante referenciadas na literatura são também desenvolvidas valendo-se de critérios metodológicos com base científica, questionamos o posicionamento teórico de Contandriopoulos et al. Tais autores concebem a avaliação como uma atividade de natureza normativa e, para seu devido enquadramento no campo científico, estabelecem a necessidade de incorporação dos elementos mencionados em sua definição de pesquisa avaliativa.

Sustentamos que a análise de programas ou de seus constituintes à luz de critérios e normas, sejam estes cientificamente estabelecidos ou não, consiste, sim, em uma atividade científica, que requer, para sua execução, determinado rigor metodológico.

Além disso, a pesquisa avaliativa, em nosso entendimento, pode perfeitamente se concentrar na análise de outros aspectos que não aqueles apontados por Contandriopoulos, desde que, concordando com Sala (1993), os instrumentos a serem utilizados estejam em consonância com a natureza do objeto a ser avaliado.

Conforme assinala Pinto (1986, apud Acurcio et al., 1991:51): "não há uma pergunta-chave ou um caminho metodológico padrão por trás de toda a avaliação de programas sociais, pois não existe uma verdade única na esfera dos fenômenos sociais. (...) Dependendo do postulado teórico assumido, encaminhar-se-á em uma ou outra direção e se selecionarão algumas dimensões para análise, em detrimento de outras. (...) O que torna científica uma avaliação não é a descoberta de uma única verdade, e sim, o esforço para verificar observações e validar o seu significado ou seus diferentes significados".

É preciso delimitar com clareza o objeto da avaliação, ou melhor, o que se pretende avaliar: um programa/serviço na íntegra ou seus elementos constituintes, ou ainda a dimensão qualidade, que se configura no âmbito das idéias e permeia tanto o programa/serviço na totalidade, quanto todos os seus elementos separadamente. Essa decisão traz profundas implicações aos rumos da pesquisa que se propõe desenvolver, já que definirá a estratégia a ser empregada na sua condução.

A constituição do campo de avaliação de serviços e programas, vale ressaltar, caracterizou-se pelo uso de pressupostos típicos do rigor positivista, cuja influência se mostra ainda presente, acompanhando a tendência histórica na administração pública do país de se privilegiarem dados quantitativos (Lobo, 1998).

Assim, predominam os estudos que não só defendem a medição estatística como "único meio de valorar alguma coisa” (Nuto \& Nations, 1999), como também concebem a avaliação como uma modalidade de pesquisa que se utiliza de métodos e técnicas específicos para confirmar ou não a relação de causalidade entre as ações de um serviço ou programa e determinados resultados:

“...conjunto de procedimentos para a coleta e análise de dados que aumentam mais a possibilidade de "provar" do que "afirmar" o valor de uma atividade social" (Suchman, 1967, apud Aguilar \& Ander-Egg, 1994:27).

“...consiste simplesmente na coleta e combinação de dados de trabalho mediante a definição de metas que proporcionam escalas comparativas ou numéricas, com o fim de justificar 1 . Os instrumentos de coleta de dados, 2 . As valorações e 3. A seleção das metas" (Scriven, 1967, apud Aguilar \& Ander-Egg, 1994:26).

“É um processo que tenta determinar o mais sistemática e objetivamente possível a relevância, efetividade e impacto das atividades, tendo em vista seus objetivos..." (ONU, 1984, apud Silver, 1992:198).

“A particularidade da avaliação de políticas públicas consiste na adoção de métodos e técnicas de pesquisa que permitam estabelecer uma relação de causalidade entre um programa $x e$ um resultado y, ou ainda, que na ausência do programa $x$, não teríamos o resultado y" (Figueiredo \& Figueiredo, 1986, apud Arretche, 1998:31).

Convém destacar que o paradigma positivista referido, tradicionalmente hegemônico em se tratando de avaliação de serviços, já começa a ser questionado (Furtado, 2001), e, segundo Deslandes (1997:104), "é possível afirmar que a avaliação de serviços de saúde ao longo dos últimos anos deixou de ser considerada um processo exclusivamente técnico...", ou se- 
ja, já se admite a subjetividade inerente a qualquer avaliação, seja ela de um fato do cotidiano, seja de um serviço ou programa em especial.

Não obstante, é possível encontrar, na literatura, algumas experiências de avaliação que não se prendem à medição e incorporam a subjetividade ao processo avaliativo (Bosi \& Affonso, 1998; Gattinara et al., 1995; Minayo et al., 1999; Nuto, 1999; Santos, 1995).

Guba \& Lincoln (1990, apud Contandriopoulos, 1997) mostram que, no estágio emergente (atual) da avaliação, pressupõe-se - para a sua realização - um processo de negociação entre os atores envolvidos na intervenção a ser avaliada. Os mesmos autores apontam também três estágios anteriores da avaliação, cuja evolução se fez possível com o desenvolvimento teórico e a acumulação do saber.

O primeiro estágio é baseado na medida (dos resultados escolares, da inteligência, da produtividade dos trabalhadores). $\mathrm{O}$ avaliador é essencialmente um técnico que tem que saber construir e saber usar os instrumentos que permitem medir os fenômenos estudados. O segundo estágio se fortalece nos anos 20 e 30 . Ele trata de identificar e descrever como os programas permitem atingir seus resultados. O terceiro estágio é baseado no julgamento. A avaliação deve permitir o julgamento de uma intervenção.

Embora possa ser válido esse esforço de sistematização, especialmente pela sugestão que faz à atualidade dos estudos que consideram a perspectiva dos atores sociais na avaliação, em alusão ao próprio caráter dinâmico do conhecimento, cabe assinalar que não nos parece suficientemente clara a idéia de passagem entre os estágios. A forma como essa transição é mencionada causa-nos a impressão de que essas etapas seriam estanques, com limites rigorosamente definidos, o que não permite vislumbrar, por conseguinte, a coexistência de modelos ou padrões de avaliação distintos ao longo da evolução histórica da pesquisa em avaliação.

Na saúde pública, a exemplo das demais áreas em que também se aplica - educação, administração, economia e serviço social, entre outras -, a avaliação de programas e serviços torna-se extremamente relevante, diferenciando-se apenas pelos contornos que assume no sentido de possibilitar uma maior compatibilidade com a natureza e especificidade do campo temático (Deslandes, 1997).

Aqui, o modelo proposto por Donabedian em 1966 (apud Aguilar \& Ander-Egg, 1994) baseado na análise de estrutura, processo e resultado - tem apresentado grande aceitabilidade e, por esse motivo, tem sido amplamente difundido. Isso se verifica em virtude de sua com- patibilidade e subseqüente utilidade para os programas de saúde, uma vez que essa abordagem contempla a lógica de funcionamento desses serviços de atendimento direto: recursos, organização, atividades, serviços e efeitos (Aguilar \& Ander-Egg, 1994).

Essa abordagem nos parece, entretanto, limitada para as demais políticas sociais. As inúmeras variações nas modalidades de intervenção que estas apresentam resultam em igualmente diversificadas dinâmicas de funcionamento, as quais, por causa dessa mesma multiplicidade, não podem ser sustentadas valendose de uma única racionalidade, em analogia ao exemplo comentado no parágrafo anterior.

Tal proposição é fundamental nas situações em que os programas ou serviços sob análise apresentarem características e configurações específicas, que os diferenciem das políticas de saúde em geral (caracterizadas pelo serviço direto de atendimento, de caráter curativo) tradicionalmente desenvolvidas.

Alguns programas encontram-se circunscritos àquela vertente de programas de saúde responsável pela promoção da saúde ou, como sugerem Akerman \& Nadanovsky (1992), responsável por “advogar saúde”. Essa diferença implica reconhecer que os modelos teóricos de avaliação da qualidade destinados a avaliar serviços curativos de atendimento não podem ser aplicados a partir de sua simples transposição ao contexto dos programas alicerçados em outros modelos de intervenção.

Além disso, é preciso entender que uma transposição dessa ordem levaria, para este último contexto, as mesmas limitações inerentes ao procedimento de avaliar um serviço a partir da fragmentação e pré-categorização de sua qualidade formal (Demo, 1999), tal como ocorre no campo dos serviços de atendimento direto, que serão comentados adiante.

\section{A qualidade de serviços e programas}

É possível identificar, na literatura, inúmeras definições de qualidade, cada qual elaborada tendo como base perspectivas particulares, que traduzem seu caráter pluridimensional.

Quando nos referimos à multidimensionalidade do termo qualidade, tomamos a liberdade de fazê-lo por dois motivos: primeiro, por entendermos que o termo qualidade assume várias dimensões ou sentidos, de natureza objetiva ou subjetiva - a título de ilustração, no Novo Dicionário da Língua Portuguesa (Ferreira, 1986), são encontrados oito conceitos sobre o termo, que variam de "propriedade ou atri- 
buto das coisas ou pessoas", portanto, passível de mensuração, a "aspecto sensível, e que não pode ser medido, das coisas" -; segundo, porque a qualidade varia de acordo com o interesse de grupos ou atores sociais, considerando-se seu papel dentro da instituição gestora e/ou de sua relação com o serviço em questão (Acurcio et. al., 1991; Akerman \& Nadanovsky, 1992; Carvalho et al., 2000; Cohen \& Franco, 1998; Furtado, 2001; Santos, 1995; Vuori, 1991). No primeiro caso, representaria o que denominamos multidimensionalidade intrínseca e, no segundo caso, o que denominamos multidimensionalidade extrínseca.

Além disso, concordamos com Demo (2000: 10), ao enfatizar que os termos quantidade e qualidade não ensejam sentidos dicotômicos, estanques, mas facetas da mesma realidade: "quantidade, para qualidade, é base e condição", enquanto que a "qualidade, por sua vez, aponta para a dimensão da intensidade".

$\mathrm{Na}$ análise do tema desenvolvimento humano, o autor identifica dois tipos de qualidade: a qualidade formal e a qualidade política. A primeira estaria relacionada a "instrumentos, formas, técnicas e métodos" diante dos desafios do desenvolvimento, em uma dimensão objetiva, mensurável (Demo, 1999:9). A outra estaria relacionada a "finalidades, valores e conteúdos", em uma dimensão subjetiva, simbólica (Demo, 2000:14). Explicita-se, assim, a qualidade valendo-se de duas dimensões distintas: a dimensão objetiva e a dimensão subjetiva.

A qualidade em sua dimensão objetiva é mensurável, e, portanto, generalizável. O mesmo não ocorre com a dimensão subjetiva. Esta última habita o espaço das vivências, das emoções, do sentimento, os quais não cabe quantificar, uma vez que expressam singularidades.

Essa classificação poderia, a nosso ver, ser muito útil no campo da saúde pública, uma vez que se observa uma dificuldade não apenas em conceituar qualidade em saúde, mas até mesmo em identificar a polissemia inerente ao termo qualidade (Nogueira, 1994; Nuto \& Nations, 1999; Serapioni, 1998).

Nesse campo específico, a qualidade dos programas e serviços é tratada - quase que exclusivamente - de maneira objetiva, quantitativa. Predominam, na literatura científica, os estudos que consideram a qualidade da saúde unicamente com base em sua dimensão formal, ou melhor, em seus componentes ou elementos, terminando-se, desta forma, por conferir um tratamento reducionista ou unidimensional à qualidade.

A Comissão Castonguay - Comissão Canadense de Inquérito sobre Saúde e Serviços So- ciais - (1970, apud Silver, 1992:195) defende que "escolher entre as possíveis definições de qualidade, nos leva a rejeitar uma parte da realidade, reduzindo o significado de qualidade a uma de suas múltiplas dimensões".

Isso talvez ocorra devido à própria complexidade inerente ao já citado caráter polissêmico do termo qualidade, que admite a subjetividade de forma tão bem demonstrada por Demo, e que se apresenta como um obstáculo epistemológico aos empreendimentos de cunho positivista, colocando em questionamento a própria racionalidade teórica que os fundamenta, considerando que o entendimento da realidade sob uma ótica que se distancie da busca exclusiva pela objetividade acaba por configurar um conflito com os pressupostos teóricos que fundamentam a perspectiva clássica de cientificidade. Conforme ressalta Nuto (1999:121), “esse conceito mantido pelos serviços de saúde resulta da apropriação da racionalidade científica moderna, em que o conhecimento biológico se superpõe a qualquer discussão e descoberta dos significantes socioculturais".

Entre os pioneiros a tentar definir a qualidade - no contexto dos serviços e programas de saúde - considerando seus componentes, temos Lee \& Jones (1933, apud Acurcio et al., 1991). Seriam eles: fundamentação científica da prática médica; prevenção; cooperação entre consumidores e prestadores de serviço; tratamento integral do indivíduo; relação estreita e contínua entre o médico e o paciente; serviços médicos integrais e coordenados; coordenação entre atenção médica e serviços sociais; acessibilidade da atenção para toda a população.

Gattinara et al. (1995) também sinalizam vários fatores que determinam a qualidade dos serviços de saúde: competência profissional (habilidades técnicas, atitudes da equipe, habilidades de comunicação); satisfação dos usuários (tratamento recebido, resultados concretos, custo, tempo); acessibilidade (cultural, social, geográfica, econômica); eficácia (normas adequadas, tecnologia apropriada, respeito às normas pela equipe); eficiência (custos, recursos, riscos).

Já Vuori (1991; também citado por Acurcio et al., 1991; Akerman \& Nadanovsky, 1992; Santos, 1995) aponta os seguintes aspectos ou dimensões da qualidade desejáveis nos serviços de saúde: efetividade (relação do impacto real com o impacto potencial numa situação ideal); eficácia (capacidade de produzir o efeito desejado); eficiência (relação entre o efeito real e os custos da produção); eqüidade (distribuição dos serviços de acordo com as necessidades de saúde objetivas e percebidas da população); 
qualidade científico-técnica (nível de aplicação do conhecimento e da tecnologia); acessibilidade (remoção de obstáculos físicos, financeiros e outros para a utilização de serviços disponíveis); adequação (suprimento de número suficiente de serviços em relação às necessidades e à demanda); aceitação (fornecimento de serviços que estão de acordo com as normas culturais, sociais e outras, e com as expectativas dos usuários em potencial).

Acurcio et al. (1991) mencionam ainda o trabalho de Gomes, que acrescenta mais duas variáveis aos componentes listados por Vuori: continuidade e comunicação eficaz médicopaciente. Continuidade refere-se ao tratamento dentro de uma perspectiva integral, em que o paciente recebe os serviços em seqüência e intervalo apropriados, enquanto comunicação eficaz médico-paciente diz respeito ao tipo de interação que pode facilitar e promover eficazmente a cooperação do paciente em relação às observações prescritas pelo médico.

É preciso entender que a identificação de todos esses componentes se deu a partir do estudo da qualidade do cuidado médico. Por isso, mais uma vez recorremos à lembrança da especificidade de determinados programas para assinalarmos que, em outro contexto, estes aspectos talvez não apresentem a mesma relevância que no contexto dos serviços de atendimento direto. Além disso, podem também ser evidenciadas outras dimensões da qualidade, igualmente relevantes, embora não exploradas devidamente pela especificidade que guardam com a natureza de certos programas, ou ainda pela preocupação, por parte dos pesquisadores, em imprimir objetividade à investigação.

A mera transposição desse modelo ao contexto dos programas comprometidos com a promoção da saúde implicaria reiterar uma posição fragmentada e reducionista, fundada em aspectos objetivos e mensuráveis da qualidade e, em geral, materializada a partir do estudo de seus componentes.

Convém também observar que análises voltadas ao estudo dos componentes, seja do cuidado médico ou de um programa de promoção da saúde, constituiriam estudos da qualidade na sua dimensão objetiva, ou seja, avaliações da qualidade formal de um programa. Já os estudos centrados na análise da dimensão subjetiva da qualidade, que buscam apreender a experiência vivencial de atores sociais que interagem com o programa ou serviço, sejam eles usuários, técnicos, gestores ou políticos, caracterizariam estudos de avaliação qualitativa de programas.

Já é possível perceber, em publicação mais recente de Donabedian (1990), a presença de elementos que sugerem a importância da subjetividade na análise da qualidade, ainda que isso não implique uma ruptura com a objetivação. Para o autor, “...la calidad de la atención a la salud debe definirse como el grado em el que los medios más deseables se utilizan para alcanzar las meyores mejoras em la salud" (Donabedian, 1990:12). Nessa perspectiva, a qualidade em saúde se refere à relação entre meios $\mathrm{e}$ fins, em que os meios compreendem o conjunto das estratégias técnicas, psicológicas e sociais de intervenção.

Acredita-se que a posição dos atores sociais que avaliam ou emitem um julgamento influencia a definição de qualidade e a relevância de determinados critérios ou componentes em detrimento de outros (Acurcio et al., 1991; Akerman \& Nadanovsky, 1992; Carvalho et al., 2000; Cohen \& Franco, 1998; Franco \& Campos, 1998; Furtado, 2001; Santos, 1995; Silver, 1992; Vuori, 1991).

Os chamados estudos antropossociais, vertente cuja metodologia é baseada nos indivíduos ou grupos sociais, desenvolveram-se principalmente a partir da década de 70 e seus eixos principais são o estudo da acessibilidade e da satisfação dos usuários (Reis et al., 1990).

Em 1983, o Relatório Griffith, publicado pelo Serviço Nacional de Saúde da Inglaterra, recomendava claramente a análise das percepções e das expectativas dos pacientes e da comunidade na avaliação de serviços públicos de saúde. A interpretação do relatório foi (não ocasionalmente) distorcida, pois a partir de então verificou-se uma grande produção de estudos baseados na satisfação do usuário, que passou a ser medida em inquéritos através de questionários (Williams, 1994).

O trecho abaixo, de autoria de Donabedian (1984, apud Oliveira, 1992:10), é ilustrativo desse pensamento: "La satisfacción del paciente és de fundamental importância como una medida de calidad de la atención, porque proporciona información sobre el éxito del provedor en alcanzar los valores y expectativas del paciente, que son asuntos sobre los que éste es la autoridad última. La medición de la satisfacción es, por lo tanto, un instrumento valioso para la investigación, administración y planeación."

As críticas a esses tipos de estudo são inúmeras. Em primeiro lugar, porque eles seriam insuficientes para avaliar a qualidade de serviços e programas (Serapioni, 1998); também não estariam contemplando as percepções e expectativas dos usuários, visto que a riqueza e complexidade das percepções e expectativas não podem ser reduzidas a meras expressões de satisfação (Williams, 1994). Tampouco esses ques- 
tionários estruturados possibilitariam acessar o fenômeno focalizado (Atkinson, 1993), eles apenas forçam o indivíduo a se expressar utilizando termos estranhos a seu vocabulário, criando assim inferências falseadas acerca de suas opiniões sobre os serviços (Williams, 1994).

Nuto \& Nations (1999:27) sustentam que " $a$ satisfação e a insatisfação estão ligadas à coerência e à incoerência que o sistema alcança entre a produção de necessidade-demanda, a oferta e a possibilidade concreta de aquisição (consumo)...”, portanto, “...o indivíduo que conseguiu ser atendido em um determinado dia, independente das horas ou dias de espera por uma vaga, fatalmente responderá que está satisfeito", confirmando, pois, a impossibilidade de analisar percepções, expectativas e opiniões valendo-se de inquéritos que se propõem a medir o "grau de satisfação" do usuário.

As avaliações não podem se restringir a pesquisas que têm como objetivo a simples obtenção de respostas numéricas. É necessário considerar a importância da subjetividade implícita ao processo de avaliar, mas, para isso, faz-se necessária a superação daquelas tendências que defendem sua utilização exclusiva para aferição de resultados ou que buscam facilitar a sua operacionalização, pois: "quality has to do with nuance, with detail, with the subtle and unique things that make a difference beyond the points on a standardized scale. Quality is what separates and falls between those points on a standardized scale (...). This is not a question of interval versus ordinal scaling, but one of meaning" (Patton, 1987:30).

As percepções dos atores sociais sobre a qualidade dos serviços são determinadas por suas experiências inerentes à vivência junto aos programas em questão. Esse entendimento permitiria compreender não apenas o significado da qualidade para os diferentes grupos, mas também as formas pelas quais esta se apresentaria ou não nesses programas.

Concordando com Silver (1992:208), reiteramos que “... não existe uma metodologia única para a avaliação de serviços; (...) a metodologia apropriada está diretamente vinculada ao que se pretende avaliar e à perspectiva do avaliador". Além disso, acreditamos que método refere-se mais a um fundamento epistemológico "que lhe dá suporte e consistência" do que a um conjunto de técnicas e instrumentos que permitem operacionalizar uma pesquisa (Oliveira, 1998:21).

Guattari (1992, apud Castiel, 1994:25) define a subjetividade como o "conjunto das condições que torna possível que instâncias individuais e/ou coletivas estejam em posição de emer- gir como território existencial auto-referencial, em adjacência ou em relação de delimitação com uma alteridade ela mesma subjetiva". Para o autor, a subjetividade se configuraria tanto na esfera individual, considerando-se o conjunto de valores e as tradições construídas com base na experiência vivida de cada indivíduo, quanto na esfera coletiva, já que abrigaria “...uma multiplicidade que se desenvolve para além do indivíduo, no socius...”, por meio da inserção particular em um determinado contexto cultural, econômico e social.

\section{Considerações finais}

A idéia de desvendar o outro lado da qualidade se nos apresenta inadiável. A dimensão subjetiva da qualidade de programas e serviços remete-nos a um terreno pouco explorado. Guarda, certamente, em seus meandros, muitos aspectos a serem desvelados, já que pertence ao plano das nuanças, do profundo, do particular.

Entendemos a premência de se considerar a participação dos atores e, principalmente, dos usuários, na avaliação. Uma investigação que pretenda desvendar um objeto de natureza qualitativa deve, obrigatoriamente, prever a utilização de uma estratégia que permita a apreensão dos sentidos dos fenômenos, e, ao mesmo tempo, respeite sua complexidade, riqueza e profundidade.

Estudos baseados nas perspectivas dos atores sociais envolvidos em programas e serviços podem ser concebidos como análises circunscritas ao campo da avaliação qualitativa, à medida que considerarem a utilização de um método científico para a captação do significado dos fenômenos focalizados. Para tanto, impõese a utilização de um método de pesquisa apropriado para a análise qualitativa de uma intervenção a partir das dimensões relevantes aos grupos de interesse (atores sociais) que interagem com um determinado programa ou serviço.

Para fundamentar e instrumentalizar o processo de desvelamento da singularidade e do sentido presente no vivido dos usuários junto a determinados programas, a tradição qualitativa se nos apresenta como um profícuo caminho metodológico.

Em síntese, essa modalidade de investigação estaria alicerçada em uma postura de busca do sentido dos fenômenos no espaço da intersubjetividade, ou melhor, no espaço do encontro entre a subjetividade que se inscreve na vivência dos informantes e na vivência do próprio pesquisador, através das compreensões e interpretações compartilhadas. 
Ao mesmo tempo, a intersubjetividade representaria o caminho da subjetividade em direção aos graus ou perspectivas da objetividade, uma vez que esta só se manifestaria a partir da subjetividade (Boemer \& Alencastre, 1996). Trata-se, portanto, de um movimento de “...colocar-se no lugar do outro” (Simões \& Souza, 1997:13).

Tal esforço de explicitação da intersubjetividade revelaria, por seu turno, a estreita imbricação da pesquisa qualitativa ancorada nessa perspectiva com o critério ético da alteridade (Guattari, 1992, apud Castiel, 1994). Derivada do latim alter, que significa outro, a alteridade, conforme nos explica Correia (1996:70), pressupõe a pessoa como "...abertura, relação, comunicabilidade, intersubjetividade...”.

A valorização da experiência vivencial dos atores sociais, concebendo-os como esse "outro" - sujeito e protagonista de um programa ou serviço, aliada à postura epistemológica de investigação pautada na intersubjetividade da relação sujeito-investigador contemplaria, duplamente, a exigência ética da alteridade na reflexão acerca dos serviços e ações governamentais, já que a alteridade, conforme assinala Correia (1996), não só evidencia o nível das relações que se estabelecem entre as pessoas, como também as relações que se estabelecem entre o homem e a vida.

\section{Referências}

ACURCIO, F. A.; CHERCHIGLIA, M. L. \& SANTOS, M. A., 1991. Avaliação de qualidade de serviços de saúde. Saúde em Debate, 33:50-53.

AGUILAR, M. J. \& ANDER-EGG, E., 1994. Avaliação de Serviços e Programas Sociais. Petrópolis: Editora Vozes.

AKERMAN, M. \& NADANOVSKY, P., 1992. Avaliação dos serviços de saúde: Avaliar o quê? Cadernos de Saúde Pública, 8:361-365.

ARRETCHE, M. T. S., 1998. Tendências no estudo sobre avaliação. In: Avaliação de Políticas Sociais (E. M. Rico, org.), pp. 29-39, São Paulo: Editora Cortez.

ATKINSON, S., 1993. Anthropology in research on the quality of health services. Cadernos de Saúde Pública, 9:283-299.

BOEMER, M. R. \& ALENCASTRE, M. B., 1996. O referencial da fenomenologia para a prática de pesquisa. Ciencia y Enfermería, 2:13-17.

BOSI, M. L. M. \& AFFONSO, K. C., 1998. Cidadania, participação popular e saúde: Com a palavra, os usuários da rede pública de serviços. Cadernos de Saúde Pública, 14:355-365.

CARVALHO, G.; ROSEMBURG, C. P. \& BURALLI, K. O., 2000. Avaliação de ações e serviços de saúde. Mundo da Saúde, 24:72-88. 
CASTIEL, L. D., 1994. O Buraco e o Avestruz: A Singularidade do Adoecer Humano. Campinas: Editora Papirus.

COHEN, E. \& FRANCO, R., 1998. Avaliação de Projetos Sociais. Petrópolis: Editora Vozes.

CONTANDRIOPOULOS, A. P.; CHAMPAGNE, F.; DENIS, J. L. \& PINEAULT, R., 1997. A avaliação na área de saúde: Conceitos e métodos. In: Avaliação em Saúde: Dos Modelos Conceituais à Prática na Análise da Implantação de Programas (Z. M. A. Hartz, org.), pp. 29-47, Rio de Janeiro: Editora Fiocruz.

CORREIA, F. A., 1996. A alteridade como critério fundamental e englobante da bioética. In: Fundamentos da Bioética (L. Pessini \& C. P. Barchifontaine, org.), pp. 68-74, São Paulo: Editora Paulus.

COTTA, T. C., 1998. Metodologias de avaliação de programas e projetos sociais: Análise de resultados e de impacto. Revista do Serviço Público, 49:105-126.

DEMO, P., 1999. Avaliação Qualitativa. São Paulo: Editora Cortez.

DEMO, P., 2000. Educação e Qualidade. Campinas: Editora Papirus.

DESLANDES, S. F., 1997. Concepções em pesquisa social: Articulações com o campo da avaliação em serviços de saúde. Cadernos de Saúde Pública, 13:103-107.

DONABEDIAN, A., 1990. Garantía y Monitoria de la Calidad de la Atención Médica: Un Texto Introductorio. México, DF: Instituto Nacional de Salud Pública.

FERREIRA, A. B. H., 1986. Novo Dicionário da Língua Portuguesa. Rio de Janeiro: Editora Nova Fronteira.

FRANCO, S. C. \& CAMPOS, G. W. S., 1998. Avaliação da qualidade de atendimento ambulatorial em pediatria em um hospital universitário. Cadernos de Saúde Pública, 14:61-70.

FURTADO, J. P., 2001. Um método construtivista para a avaliação em saúde. Ciência \& Saúde Coletiva, 6:165-181.

GATTINARA, B. C.; IBACACHE, J.; PUENTE, C.; GIACONI, J. \& CAPRARA, A., 1995. Percepción de la comunidad acerca de la calidad de los servicios de salud públicos en los distritos Norte e Ichilo, Bolivia. Cadernos de Saúde Pública, 11:425-438.

LOBO, T., 1998. Avaliação de processos e impactos em programas sociais: Algumas questões para reflexão. In: Avaliação de Políticas Sociais (E. M. Rico, org.), pp. 75-84, São Paulo: Editora Cortez.

MINAYO, M. C. S.; SOUZA, E. R.; ASSIS, S. G.; CRUZ NETO, O.; DESLANDES, S. F. \& SILVA, C. M. P. F., 1999. Avaliação dos Centros de Orientação e Apoio Sorológico/CTA/Coas da Região Nordeste do Brasil. Cadernos de Saúde Pública, 15:355-367.

NOGUEIRA, R. P., 1994. Perspectivas da Qualidade em Saúde. Rio de Janeiro: Editora Qualitymark.
NUTO, S. S., 1999. Avaliação Cultural do Serviço de Saúde Bucal de Beberibe - CE: A Vez e a Voz do Usuário. Dissertação de Mestrado, Fortaleza: Faculdade de Medicina, Universidade Federal do Ceará.

NUTO, S. S. \& NATIONS, M. K., 1999. Avaliação qualitativa dos serviços como processo de construção de cidadania. Ação Coletiva, 2:25-29.

OLIVEIRA, C. G., 1992. Análise do Conceito de Satisfação do Usuário na Obra de Donabedian. Rio de Janeiro: Editora UERJ.

OLIVEIRA, P. S., 1998. Caminhos de construção das ciências humanas. In: Metodologia das Ciências Humanas (P. S. Oliveira, org.), pp. 17-26, São Paulo: Editora UNESP.

PATTON, M. Q., 1987. How to Use Qualitative Methods in Evaluation. Newbury Park: Sage Publications.

REIS, E. J. F. B.; SANTOS, F. P.; CAMPOS, F. E.; ACÚRCIO, F. A.; LEITE, M. T. T.; LEITE, M. L. C.; CHERCHIGLIA, M. L. \& SANTOS, M. A., 1990. Avaliação da qualidade dos serviços de saúde: Notas bibliográficas. Cadernos de Saúde Pública, 6:50-61.

RUS-PEREZ, J. R., 1998. Avaliação do processo de implementação: Algumas questões metodológicas. In: Avaliação de Políticas Sociais (E. M. Rico, org.), pp. 65-73, São Paulo: Editora Cortez.

SALA, A., 1993. A avaliação de programas de saúde. In: Programação em Saúde Hoje (L. B. Schraiber, org.), pp. 117-138, São Paulo: Editora Hucitec.

SANTOS, M. P., 1995. Avaliação da qualidade dos serviços públicos de atenção à saúde da criança sob a ótica do usuário. Revista Brasileira de Enfermagem, 48:109-119.

SERAPIONI, M., 1998. Avaliação da Qualidade em Saúde: A Contribuição da Sociologia da Saúde para a Superação da Polarização entre a Visão dos Usuários e a Perspectiva dos Profissionais de Saú$d e$. Fortaleza: Escola de Saúde Pública do Ceará. (mimeo.)

SILVA, L. M. V. \& FORMIGLI, V. L. A., 1994. Avaliação em saúde: Limites e perspectivas. Cadernos de Saúde Pública, 10:80-91.

SILVER, L., 1992. Aspectos metodológicos em avaliação dos serviços de saúde. In: Planejamento Criativo: Novos Desafios em Políticas de Saúde (E. Gallo,org.), pp. 195-209, Rio de Janeiro: Editora Relume-Dumará.

SIMÕES, S. M. F. \& SOUZA, I. E. O., 1997. Um caminhar na aproximação da entrevista fenomenológica. $R e-$ vista Latino-Americana de Enfermagem, 5:13-17.

VUORI, H., 1991. A qualidade da saúde. Divulgação em Saúde Para Debate, 3:17-24.

WILLIAMS, B., 1994. Pacient satisfaction: A valid concept? Social Science and Medicine, 38:509-516.

Recebido em 13 de julho de 2001

Versão final reapresentada em 28 de novembro de 2001 Aprovado em 1 de março de 2002 